\title{
Acute Stress Contributes to Individual Differences in Pain and Pain-Related Brain Activity in Healthy and Chronic Pain Patients
}

\author{
Etienne Vachon-Presseau, ${ }^{1,2}$ Marc-Oliver Martel, ${ }^{3}$ Mathieu Roy, ${ }^{4}$ Etienne Caron, ${ }^{2,5}$ Geneviève Albouy, ${ }^{2}$ \\ Marie-France Marin, ${ }^{6}$ Isabelle Plante, ${ }^{7}$ Michael J. Sullivan, ${ }^{8}$ Sonia J. Lupien, ${ }^{6}$ and Pierre Rainville ${ }^{2,5,9}$ \\ ${ }^{1}$ Département de Psychologie, Université de Montréal, Montreal, Quebec, Canada H3C 3J7, ${ }^{2}$ Centre de Recherche de l'Institut Universitaire de Gériatrie de \\ Montréal (CRIUGM), Montreal, Quebec, Canada H32 1W4, ${ }^{3}$ Department of Anesthesiology, Harvard Medical School, Boston, Massachusetts 02115, \\ ${ }^{4}$ Department of Psychology, University of Colorado, Boulder, Colorado 80309, ${ }^{5}$ Department of Stomatology, Faculty of Dentistry, Université de Montréal, \\ Montreal, Quebec, Canada H3C 3J7, ${ }^{6}$ Louis-H. Lafontaine Hospital, Montreal, Quebec, Canada H1N 3M5, 7Département de l'Éducation, Université du \\ Québec à Montréal, Montreal, Quebec, Canada H3C 3P8, ${ }^{8}$ Department of Psychology, McGill University, Montreal, Quebec, Canada H3A 1X1, and 9 $\mathrm{Groupe}$ \\ de Recherche sur le Système Nerveux Central (GRSNC), Université de Montréal, Montreal, Quebec, Canada H3C 3P8
}

Individual differences in pain sensitivity and reactivity are well recognized but the underlying mechanisms are likely to be diverse. The phenomenon of stress-induced analgesia is well documented in animal research and individual variability in the stress response in humans may produce corresponding changes in pain. We assessed the magnitude of the acute stress response of 16 chronic back pain (CBP) patients and 18 healthy individuals exposed to noxious thermal stimulations administered in a functional magnetic resonance imaging experiment and tested its possible contribution to individual differences in pain perception. The temperature of the noxious stimulations was determined individually to control for differences in pain sensitivity. The two groups showed similar significant increases in reactive cortisol across the scanning session when compared with their basal levels collected over 7 consecutive days, suggesting normal hypothalamic-pituitary-adrenal axis reactivity to painful stressors in CBP patients. Critically, after controlling for any effect of group and stimulus temperature, individuals with stronger cortisol responses reported less pain unpleasantness and showed reduced blood oxygenation level-dependent activation in nucleus accumbens at the stimulus onset and in the anterior mid-cingulate cortex (aMCC), the primary somatosensory cortex, and the posterior insula. Mediation analyses indicated that pain-related activity in the aMCC mediated the relationship between the reactive cortisol response and the pain unpleasantness. Psychophysiological interaction analysis further revealed that higher stress reactivity was associated with reduced functional connectivity between the aMCC and the brainstem. These findings suggest that acute stress modulates pain in humans and contributes to individual variability in pain affect and pain-related brain activity.

\section{Introduction}

Individual differences in pain sensitivity are a robust phenomenon that origins from genetic and environmental factors (Chen et al., 1989; Dionne et al., 2005; Nielsen et al., 2009). Brain-imaging studies have shown that the subjective pain experience is reflected by the individual brain activity in several targets of the spinothalamo-cortical pathways (Bornhövd et al., 2002; Coghill et al., 2003). For instance, Coghill et al. (2003) showed that individuals reporting higher heat pain sensitivity during a psychophysics experiment also displayed stronger brain activity in the primary

\footnotetext{
Received Sept. 26, 2012; revised Dec. 17, 2012; accepted Feb. 14, 2013.

Author contributions: E.V.-P., M.-F.M., M.J.S., S.J.L., and P.R. designed research; E.V.-P., M.-O.M., M.R., and E.C. performed research; E.V.-P., M.R., G.A., I.P., and P.R. analyzed data; E.V.-P., M.-F.M., and P.R. wrote the paper

This work was supported by grants from the Fonds de Recherche Québec-Santé (FRQS; P.R. and M.J.S.) and from the Canadian Institute for Health Research (CIHR; P.R.). During this project, E.V.P. received doctoral scholarships from the CIHR and FRQS. We thank Carollyn Hurst and André Cyr for their help in MRI data acquisition, and Sylvia Villeneuve for her scientific advices and support.

Correspondence should be addressed to Etienne Vachon-Presseau, Centre de Recherche de I'Institut Universitaire de Gériatrie de Montréal, 4545 Chemin Queen Mary, Montréal, QC H3W 1W4. E-mail: etienne.vachon-presseau@umontreal.ca.

DOI:10.1523/JNEUROSCI.4584-12.2013

Copyright (C) 2013 the authors $\quad 0270-6474 / 13 / 336826-08 \$ 15.00 / 0$
}

somatosensory cortex, the anterior cingulate cortex, and the prefrontal cortex when compared with less sensitive individuals. A better understanding of the causes of interindividual differences in pain sensitivity and pain-related neural responses in healthy and chronic pain patients would be crucial for improving the evaluation and the treatment of pain (Nielsen et al., 2009). One factor likely to explain variability in the brain response to noxious stimuli and in the perception of pain is the acute stress response. This is critical because both noxious stimuli and magnetic resonance imaging (MRI) context have strong intrinsic values as physical/psychological stressors (Friedman et al., 1967; Tessner et al., 2006) and acute stress responses are likely to influence the neurophysiological mechanisms underlying the perception of pain.

One of the adaptive consequences of acute stress on the organism is the inhibition of the pain system (Madden et al., 1977; Willer et al., 1981), which partly results from descending inhibition of the spinal cord (Akil et al., 1986; Hohmann et al., 2005; Butler and Finn, 2009). While animal studies mainly focused on brainstem and spinal regulation of the stress-induced analgesia, one should also expect that such inhibitory systems would in turn reduce the nociceptive activation of ascending pathways reaching 
diencephalic and telencephalic targets and should thereby reduce the cerebral pain response. In humans, recent findings indicate that acute stress response involves large-scale reorganization of the brain networks underlying the regulation of vigilance and sensory processing, including the anterior mid-cingulate cortex (aMCC), the frontoinsular cortex and subcortical regions (Hermans et al., 2011). These findings suggest that acute stress is likely to regulate the pain-related brain responses and the perception of pain.

In this study, we examined individual differences in brain responses to painful stimuli as a function of the acute stress response measured by the salivary level of cortisol in chronic back pain (CBP) patients and healthy volunteers. We first hypothesized that the administration of painful thermal stimulations in the MRI context would increase the levels of cortisol. We further examined if CBP impacts the cortisol reactivity to pain or the pain-related brain activity. We secondly hypothesized that individuals with stronger reactive cortisol responses during the imaging study would report less pain and would show less painrelated brain activity in regions referred to as the "pain matrix" (Apkarian et al., 2005). Mediation analyses were last used to determine whether the pain response in these cortical structures mediated the expected inverse relation between the reactive cortisol levels and the pain ratings.

\section{Materials and Methods}

Participants. Forty-two participants (21 idiopathic CBP patients and 21 Controls) were recruited through local pain treatment centers and newspaper advertisements in Montreal and gave written informed consent to participate to this study. The age of the CBP patients ( 11 women) ranged from 23-49 years old (mean: 36 years old) and was matched in healthy controls ( 10 women; $21-53$ years old; mean 36 years old). Patients reported having chronic idiopathic low back pain for at least 6 months. Chronic pain severity was assessed with the McGill pain questionnaire (Melzack, 1975).

Eight participants had to be excluded because of head movement exceeding $3.5 \mathrm{~mm}(n=4)$, abnormally high baseline cortisol $(n=1)$, depressive mood (Beck Depression Inventory score $>17 ; n=1$ ) and because of technical problems during the scanning session $(n=2)$. The final sample includes 16 CBP and 18 controls.

Salivary cortisol. Cortisol is the end product of the hypothalamicpituitary-adrenal (HPA) axis activation that can be used as a biomarker of the stress response. Salivary cortisol is mostly used over the plasma cortisol because it is a noninvasive sampling technique (Kirschbaum and Hellhammer, 1994). Saliva samples were obtained using the passive drool method. Participants were asked to fill a small plastic vial with $10 \mathrm{~mm}$ of pure saliva using a straw. The samples were then maintained at $-20^{\circ} \mathrm{C}$ until the time of cortisol concentration determination. Analyses were performed at the Centre for Studies on Human Stress (Douglas Mental Health Institute, Montreal Site, Canada; www.humanstress.ca) using an Enzyme Immunoassay kit from Salimetrics.

Figure 1 presents the timing at which the salivary samples were collected. Each participant was asked to collect salivary samples through the day of the scanning experiment to evaluate the effect of acute stress on cerebral pain processing. All participants were tested in the afternoon to control for circadian fluctuations of cortisol. On the day of the experiment, four samples were collected: (1) at the arrival of the participant to the lab (between 11:40 A.M. and 1:52 P.M.; mean time 1:01 P.M.), (2) before entering the scan (between 1:10 and 3:43 P.M.; mean time 2:37 P.M.), (3) immediately after getting out of the scan (between 3:05 and 5:19 P.M.; mean time 4:18 P.M.), and (4) immediately before returning home (between 4:20 and 6:25 P.M.; mean time 5:36 P.M.).

Participants were also instructed to collect five samples per day during 7 consecutive days starting the day after the scanning experiment. These samples were used to measure the basal levels of cortisol and its association with chronic pain (Vachon-Presseau et al., 2013). A linear equation predicting the expected basal levels of cortisol between 12:00 P.M. and 4:00 P.M. was calculated for each participant. This permitted us to determine the normal level of cortisol expected at the time when the reactive salivary samples were collected during the day of the scanning experiment. The individual basal levels of cortisol were then subtracted from the reactive levels of cortisol to control the diurnal variations and the individual variation of basal cortisol secretion. An area under the curve with respect to the ground (AUCg) was calculated as follows (Pruessner et al., 2003):

$$
\begin{aligned}
\text { AUCg }=\frac{(\text { sample } 3+\text { sample } 2) \times t 2}{2} & \\
& +\frac{(\text { sample } 4+\text { sample } 3) \times t 3}{2}
\end{aligned}
$$

Here, $t 2$ and $t 3$ represent the average time between the measurements. This index quantified the global cortisol increase induced by the noxious stimulations administered in the functional MRI (fMRI) context when taking into account the individual curve of basal cortisol. The individual AUCg values were used to evaluate the influence of acute stress on pain perception and pain-related brain processing.

Controlling for potential confounds. The Beck Depression Inventory, second edition (BDI II; Beck et al., 1996) was administered to ensure that no participant included in the final sample was in a depressive mood. The $\mathrm{BDI}$ is a 21 items self-report questionnaire that assessed the depressive symptoms experienced during the last 2 weeks. The mean score on the BDI was higher in the CBP $(8.3 \pm 6.5)$ than in the controls $(2.9 \pm 3.5 ; p=$ 0.006 ) but indicated minimal depressive moods (all scores $\leq 17$ ).

The patients' medication used during $60 \mathrm{~d}$ prior to the experiment was also documented. Six CBP patients reported having used the muscle relaxant, ibuprofen, nonsteroidal anti-inflammatory drugs, or serotoninnorepinephrine reuptake inhibitors to control their pain. Other forms of medications were used for thyroid regulation ( $n=1 \mathrm{CBP}$ and 1 control), asthma or allergies ( $n=2$ controls), high arterial pressure $(n=1 \mathrm{CBP})$, and anxiety ( $n=1$ control). Eight $\mathrm{CPB}$ patients and 14 controls were medication free. Additional analysis confirmed that ruling out the effect of medication by covariance did not change the results of the cortisol analyses, suggesting that the medication did not contribute to the findings of the present study.

Experimental procedure and material. Psychophysical intensity estimations were conducted using the method of constant stimuli in each participant in a pre-experimental session to determine the temperature that was perceived as strongly painful. A computer-controlled thermal stimulator-induced pain with a $3 \times 3 \mathrm{~cm}^{2}$ contact probe (Medoc TSA2001; Medoc) attached to the left lower leg. Baseline temperature always remained at $38^{\circ} \mathrm{C}$. The pain threshold was determined by asking subjects to adjust the stimulus using the buttons of a computer mouse to increase or decrease the temperature in steps of $0.1^{\circ} \mathrm{C}$ until the stimulus corresponded to the smallest intensity considered painful. This procedure was performed four consecutive times and the average temperature of the three last stimulations was used as the individual pain threshold. Then, $129 \mathrm{~s}$ heat stimuli ( $2 \mathrm{~s}$ ramps and $5 \mathrm{~s}$ plateau) were administered with temperatures of $-2,-1,0,+1,+2$, and $+3^{\circ} \mathrm{C}$ below/above the individual pain threshold. These were applied in a predetermined pseudorandom order. After each stimulus, participants were asked to evaluate if the stimulation was warm or painful and provide self-report ratings of the intensity of the stimulus using a verbal numerical report ranging between " 0 , no pain" to " 100 , extreme pain" or " 0 , no sensation" to " 100 , very warm." The ratings at each temperature were averaged and plotted against the physical stimulus level to create a psychophysical intensity curve. The temperature at the mean pain intensity rating of 75 was selected to induce pain in the MRI experiment. The temperature required to produce strong pain was comparable between the two groups (Healthy $48.5 \pm 1.2^{\circ} \mathrm{C}$ and $\mathrm{CBP}$ patients $48.1 \pm 1.3^{\circ} \mathrm{C} ; p=0.75$ ). The temperature at a psychophysical warm intensity score of 50 was selected to induce the control condition (Healthy $43.2 \pm 1.0^{\circ} \mathrm{C}$ and $\mathrm{CBP}$ patients $42.8 \pm 0.7^{\circ} \mathrm{C}$; $p=0.12$ ).

The MRI experiment consisted of two runs of thermal stimuli applied to the lower right leg of the participants that were followed by two sepa- 
rate runs during which the participant observed images displaying pain-evoking or neutral images. The data regarding painevoking or neutral images were presented previously (Vachon-Presseau et al., 2012). In two functional scans, 16 nonpainful and 16 painful thermal stimuli were applied in a pseudorandom order to the left lower leg. The rate of temperature increase from baseline was adjusted individually to reach the target temperature in $2 \mathrm{~s}$, and remained at a plateau for $5 \mathrm{~s}$ before returning to baseline in $2 \mathrm{~s}$. A long intertrial interval (18-25s) was used to prevent sensitization and to allow subjects to rate each stimulus. After each stimulus, the participant indicated if he perceived the thermal stimuli as warm or painful by using the index and middle finger keys of an MRI-compatible response box. If the stimulation was categorized as painful, the participant was asked to successively rate the intensity and the unpleasantness of the painful experience on computerized visual analog scales (VAS) displayed using E-Prime (Psychology Software Tools; http://www. pstnet.com) on a screen located at the headend of the scanner and viewed via a mirror. The scales were presented during $12 \mathrm{~s}$ each and labeled with the verbal anchor "no pain" or "not unpleasant" at 0 (left extremity) and "extreme pain" or "extremely unpleasant" at 100 (right extremity). If the stimulation was evaluated as warm, a VAS ranging from 0 , no sensation to 100 , very warm was presented during $18 \mathrm{~s}$. The ratings were produced by moving a cursor using the index and middle finger of the right hand and were recorded in E-prime.

fMRI image acquisition and analyses. Imaging was performed on a $3.0 \mathrm{~T}$ whole-body scanner (Siemens TRIO), using a 12-channel head coil, at the Centre de Recherche de l'Institut Universitaire de Gériatrie de Montréal in Montréal, Quebec, Canada. Blood oxygenation level-dependent (BOLD) signal was acquired using a standard $\mathrm{T} 2{ }^{*}$-weighted gradient-echo EPI sequence $\left(\mathrm{TR}=3000 \mathrm{~ms}, \mathrm{TE}=30 \mathrm{~ms}\right.$; flip angle $=90^{\circ}$; matrix; FOV $=$ $220 \times 220 \mathrm{~mm}^{2} ; 227$ volumes; 40 interleaved, axial slices per whole-brain volume at $3.4 \mathrm{~mm}$ thickness; in-plane resolution of $3.4 \times 3.4 \mathrm{~mm}$ for isotropic voxels). Structural images were acquired using a high-resolution, T1-weighted MPRAGE sequence $(\mathrm{TR}=2300 \mathrm{~ms} ; \mathrm{TE}=2.99 \mathrm{~ms}$; flip angle $=$ $9^{\circ} ; \mathrm{FOV}=256 \mathrm{~mm}$; matrix $=256 \times 256 ; 1 \times 1 \times 1.2 \mathrm{~mm}$ voxels; 160 slices per whole-brain volume). All data preprocessing and analysis was done using SPM 8 (Statistical Parametric Mapping, Version 8; Wellcome Department of Imaging Neuroscience, London, UK) executed in MATLAB 7.8. (Mathworks). Off-line preprocessing of functional images included slice-time correction, motion correction and realignment, coregistration of each subject's functional and anatomical volumes, spatial normalization (MNI space), spatial smoothing $(8 \mathrm{~mm}$ full-width at half-maximum (FWHM) Gaussian kernel), temporal smoothing with a high-pass filter to remove low-frequency noise (cutoff $=128 \mathrm{~s}$ ), and correction for autocorrelation using a first-order autoregressive model (AR1).

The analysis of fMRI data was based on a mixed effects model conducted in two serial steps accounting for fixed and random effects. For the fixed effect, a general linear model estimated changes in brain regional responses for each subject. The noxious and the warm stimuli and their subevents were modeled using the following six regressors: Warm $_{\text {ramp-up, Warm, Warm }}$ rating, Pain ${ }_{\text {ramp-up, Pain, and Pain }}$ ratings. The ramp-up consisted of $2 \mathrm{~s}$ during which the temperature rose to the target level, and the Warm/Pain events consisted of the $5 \mathrm{~s}$ plateau and the $2 \mathrm{~s}$ ramp-down during which subjects typically experience some pain before the stimulus has reached baseline temperature. For each trial type, a given item was modeled as a delta function representing its onset and duration. The ensuing vector was convolved with the canonical hemodynamic response function, and used as a regressor in the individual design matrix. Movement parameters estimated during realignment (translations in $x, y$, and $z$ directions and rotations around $x$-, $y$-, and $z$-axes) and constant vector were also included in the matrix as a variable of no interest.

Linear contrasts tested the main effect of pain by subtracting warmrelated brain activity from the pain-related brain activity [Pain vs Warm] to control for nonspecific neural response to thermal sensory input. This linear contrast and the main effect of Pain ${ }_{\text {ramp-up }}$ generated statistical parametric maps $[\operatorname{SPM}(\mathrm{T})]$. These summary statistical images were then further spatially smoothed (Gaussian kernel $6 \mathrm{~mm}$ FWHM) and entered in a second-level analysis, corresponding to a random effects model and accounting for intersubject variance.

The random effects model was first used to perform two-sample $t$ tests comparing the main effect of Pain ${ }_{\text {ramp-up }}$ and the contrast [Pain vs Warm] between the groups. This permitted us to determine whether neural activity differed in CBP patients. As no significant group effect was observed in these contrasts, the data from all subjects were used in the following analyses to test the relation between the cortisol response and pain-evoked BOLD responses. To this end, the individual within-

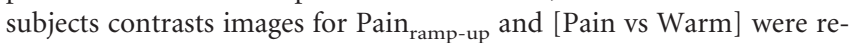
gressed against the individual AUCg. As a conservative measure, these analyses included covariates controlling for the group and the baseline individual pain sensitivity as determined by the temperature required to produce strong pain in the prescanning session. The inclusion of these covariates did not affect the main findings reported here. 
The resulting set of voxel values for each contrast constituted a map of the $t$ statistic $[\operatorname{SPM}(\mathrm{T})]$ that was thresholded at an uncorrected $p<0.001$. Statistical inferences were based on a familywise error correction $(p<$ 0.05 ) over regions of interest (ROI). Previous studies have shown that pain involves a biphasic BOLD-signal change (Becerra et al., 2001) corresponding to an early response coding the motivational response to the noxious stimulation in the nucleus accumbens (NAc; Baliki et al., 2010) and a later response corresponding to the peak of the conscious pain experience that is coded in the primary and secondary somatosensory cortices ( $\mathrm{S} 1$ and $\mathrm{S} 2$ ), the aMCC, and the posterior insula (pINS) (Apkarian et al., 2005). The NAc, the cingulate cortex, the post central gyrus, and bilateral insula were therefore defined anatomically using the WFU PickAtlas software toolbox (Maldjian et al., 2003) and used as a priori determined ROIs.

Mediation analysis. A mediation analysis is a statistical procedure used to examine the direct and indirect (mediating) links between variables to test a given theoretical model empirically (Lleras, 2005). To evaluate the mediation hypothesis, bootstrap analysis simulating 2000 samples were performed with Amos statistical software package (http://www-01.ibm. com/software/analytics/spss/products/statistics/amos/). The bootstrapping method tests whether the indirect effect of a predictor variable on the outcome variable is significant. Here, we first extracted the mean parameter estimates in a small sphere of $3 \mathrm{~mm}$ radius around the peak of activation that negatively correlated with the AUCg in the aMCC, the pINS, and S1 and secondly tested if the brain activity in each one of these regions indirectly predicted the pain unpleasantness ratings.

Functional connectivity. Psychophysiological interaction (PPI) analyses (Friston et al., 1997) were computed to identify brain regions involved in descending modulation showing a significant change in functional connectivity with the aMCC (see below) in proportion to the acute stress response. New linear models were generated at the individual level, using three regressors. The first represented the condition [Pain vs Warm], the second the activity extracted in the reference area, and the third the interaction of interest between the first (psychological) and the second (physiological) regressors. The individual within-subject contrast was then regressed against the individual AUCg to determine functional connectivity within an ROI delineating the midbrain, the pons, and the medulla. This analysis indicates the modulation effect of the stress response on the strength of the functional connectivity between the aMCC and the brainstem ROI.

\section{Results}

\section{Warm and pain perception}

Baseline individual differences in thermal sensitivity were controlled using a stimulus-adjustment procedure. The warm stimulation was rated as slightly to moderately intense on the VAS during scanning in both groups (Healthy $31.2 \pm 16.1$, and CPB patients $28.1 \pm 20.6 ; p=0.61$ ). The noxious thermal stimulations also induced similar levels of pain during the scanning session, as confirmed by the ratings of pain intensity (mean $\pm \mathrm{SD}$ : Healthy $75.0 \pm 9.4$; CBP patients $77.5 \pm 13.7 ; p=0.54$ ) and unpleasantness (Healthy $72.7 \pm 8.7$ and CBP patients $75.8 \pm$ $14.7 ; p=0.46$ ). Multilevel linear modeling was used to determine whether differences in cortisol were confounded with possible individual differences in sensitization/habituation. The results show that there was no global sensitization/habituation effect across the group and that individual differences in cortisol reactivity were not related with temporal changes in pain intensity or unpleasantness ( $p$ 's $>$ 0.65 ). This confirmed that we adequately controlled for baseline individual sensitivity differences by selecting stimuli that produced the target levels of pain and that the paradigm produced reliable pain experiences throughout the experiment.

\section{Cortisol levels increase during the scanning experiment}

Cortisol levels measured from the samples collected at the arrival of the participant to the lab (Sample 1), before getting in the

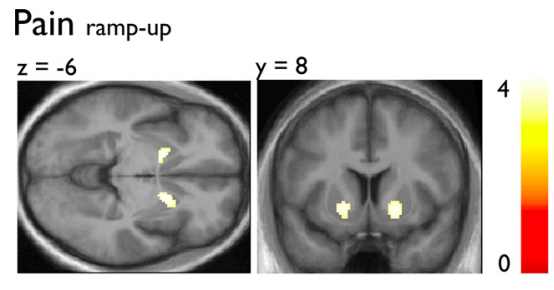

Pain Vs Warm

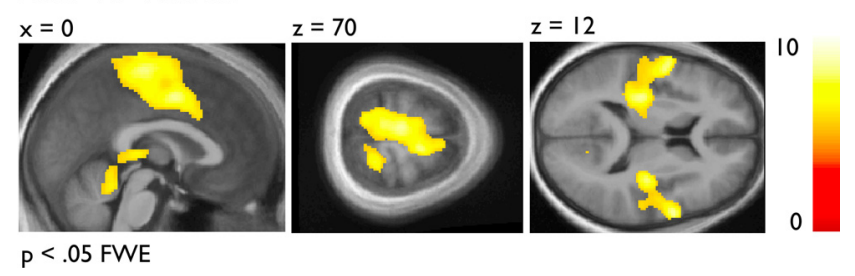

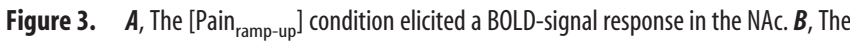
[Pain vs Warm] contrast elicited a BOLD-signal response in pain-related brain regions. Note that both analyses controlled for the group and the individual temperature used to elicit strong heat pain (see Materials and Methods). Functional data are shown over the mean structural image of all participants (displayed at $p<0.001$ uncorrected) unless otherwise specified.

Table 1. Pain-related brain activations

\begin{tabular}{lllll}
\hline & \multicolumn{2}{l}{ MNI coordinates } & & Local peak \\
\cline { 2 - 4 } Brain area & $x$ & $y$ & $z$ & $z$-value \\
\hline
\end{tabular}

Peaks of activation for the main effect [Pain ${ }_{\text {ramp-up }}$ ] $(p<0.001$ unc.)

Anterior insula

Nucleus accumbens

$\begin{array}{rrrr}34 & 22 & 8 & 3.25 \\ -30 & 18 & 10 & 3.24 \\ 18 & 8 & -6 & 3.59 \\ -18 & 6 & -4 & 3.58\end{array}$

Peaks of activation for the [Pain vs Warm] contrast $(p<0.05$ FWE $)$

Pre central gyrus

$\begin{array}{rrrr}60 & 4 & 8 & 6.67\end{array}$

aMCC

Superior frontal gyrus

Posterior insula

$\begin{array}{rrrr}-56 & -2 & 6 & 6.31 \\ 6 & -4 & 44 & 6.41\end{array}$

Thalamus

$\begin{array}{llll}10 & -6 & 68 & 5.94\end{array}$

$\begin{array}{llll}36 & -16 & 16 & 6.20\end{array}$

$\begin{array}{llll}-32 & -22 & 18 & 6.30\end{array}$

$\begin{array}{llll}14 & -20 & -2 & 4.93\end{array}$

Paracentral lobule

Lateral operculum

$\begin{array}{llll}-10 & -24 & -6 & 5.77\end{array}$

$\begin{array}{llll}6 & -22 & 66 & 6.40\end{array}$

$\begin{array}{rrrr}56 & -24 & 22 & 6.22\end{array}$

$\begin{array}{lllll}\text { Post central gyrus } & -10 & -36 & 64 & 6.22\end{array}$

$\begin{array}{rrrr}20 & -42 & 68 & 5.54 \\ 4 & -44 & -18 & 5.19\end{array}$

$\begin{array}{llll}36 & -44 & -34 & 5.90\end{array}$

The peak of activation during the early phase of the pain response (top; Pain ramp-up ) when using a threshold of $p<$ 0.001 uncorrected and during the pain response (bottom; Pain vs Warm) when using a threshold of $p<0.05$ after correcting for FWE on the whole brain.

scanner (Sample 2) and before returning home (Sample 4) were almost identical to basal levels estimated from the diurnal curves obtained in the following $7 \mathrm{~d}$. The sample collected when the participant got out the scanner (Sample 3), however, reveals a clear increase in levels of cortisol in both groups (one-tailed $t$ test Fig. $1 A$; Healthy $t_{(17)}=2.4 ; p=0.014$; Fig. $1 B$; CBP patients $\left.t_{(15)}=2.1 ; p=0.028\right)$. Computing the AUCg by subtracting the individual curve of basal cortisol allowed quantifying this acute response. No differences in the mean AUCg were observed between the two groups, suggesting normal HPA axis reactivity to the stressor in the CPB patients (Healthy 7.1 \pm 13.5 ; CBP patients $6.5 \pm 17.0 ; p=0.19)$. This index of cortisol reactivity was used as a between-subject variable in the fMRI analyses (Fig. 2). 


\section{Pain-related brain activation}

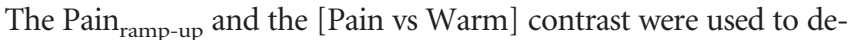
termine brain regions involved in the onset phase and in pain-related processes. Figure 3 shows the combined pain-related brain activations. Figure $3 A$ confirms that the NAc had increasing activity during the Pain ${ }_{\text {ramp-up }}$ (see Table 1 ). Figure $3 B$ revealed that the [Pain vs Warm] contrast elicited peaks of activity in several regions often referred to as the "pain matrix" that included the thalamus, the primary sensorimotor cortices, the lateral parietal operculum, the insular cortex and the aMCC (Apkarian et al., 2005). No significant group difference was found for the pain effects ( $p$ 's $>0.001$ uncorrected).

\section{Reduced pain in individuals showing a stronger stress response}

To investigate the relationship between acute stress and brain activity, the interindividual AUCg was entered as a regressor pre-

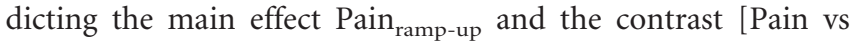
Warm] in predetermined ROI. Figures 4 and $5 A$ show that the early response of the $\operatorname{NAc}(x, y, z 16,6,-10)$ and the pain response in the aMCC, $\mathrm{S} 1$ (matching with the leg representation), and the pINS correlated negatively with the AUCg (see Table 2). Complementary analysis shown in the figures indicated that both groups contributed to these effects. Importantly, no brain activation correlated positively with the AUCg, in the onset phase or the pain response, even at a less stringent threshold of $p$ uncorrected $<$ 0.001 . The results also show that the individual differences in pain unpleasantness were inversely related to AUCg $(r=-0.39 ; p=$ $0.03)$. A similar negative correlation between the AUCg and pain intensity ratings was also observed but failed to reach significance $(r=-0.29 ; p=0.11)$. Together, these effects indicate that healthy and CBP patients showing a stronger stress response during scanning also showed reduced painrelated brain activity and reported less pain unpleasantness.

\section{The relation between acute stress and} pain is mediated by brain activity in the aMCC

Bootstrap analyses were used to determine whether an indirect effect of brain activity in the aMCC, the S1, and the pINS could mediate the direct relationship between the AUCg and pain unpleasantness. Figure $5 B$ shows that the relation between the AUCg and pain unpleasantness was fully mediated by the brain activity in the aMCC $(\mathrm{b}=-0.31$, $p=0.01 ; 95 \% \mathrm{CI}:-0.63$ to -0.08$)$, but not in the S1 or the pINS ( $p$ 's $>0.43$ ). These results support a key role of the aMCC in the regulation of pain by stress. Finally, a PPI analysis revealed that individuals with stronger stress responses showed reduced functional connectivity between pain-related activity in the aMCC and the brainstem (Table 3 and Fig. 6). This suggests that stress-related analgesia is associated with reduced brain activity in the aMCC and functional connectivity with the brainstem regions involved in descending modulation mechanisms.

\section{Regression of $\mathrm{AUCg}$ with Pain ramp-up}
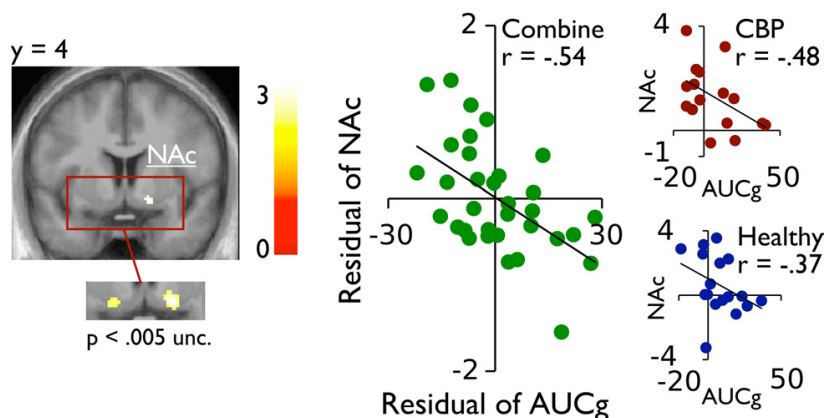

Figure 4. The AUCg of the reactive cortisol response negatively correlated with the BOLD signal response in the $\mathrm{NAc}(x, y, z 16,6,-10)$. The scatter plot (green) represents the residual of the mean parameter estimates (a.u.) in the right NAc against the residual $A U C g$ of the reactive cortisol, after controlling for the group and the stimulus temperature (see Materials and Methods). Additional scatter plots represent the mean parameter estimates (a.u.) in the NAc against the AUCg for reactive cortisol in each CBP patient (red) and healthy individuals (blue) separately. Functional data are shown over the mean structural image of all participants (displayed at $p<$ 0.001 uncorrected unless otherwise specified).
A Regression of AUCg with [Pain Vs Warm] B Mediation between AUCg and pain
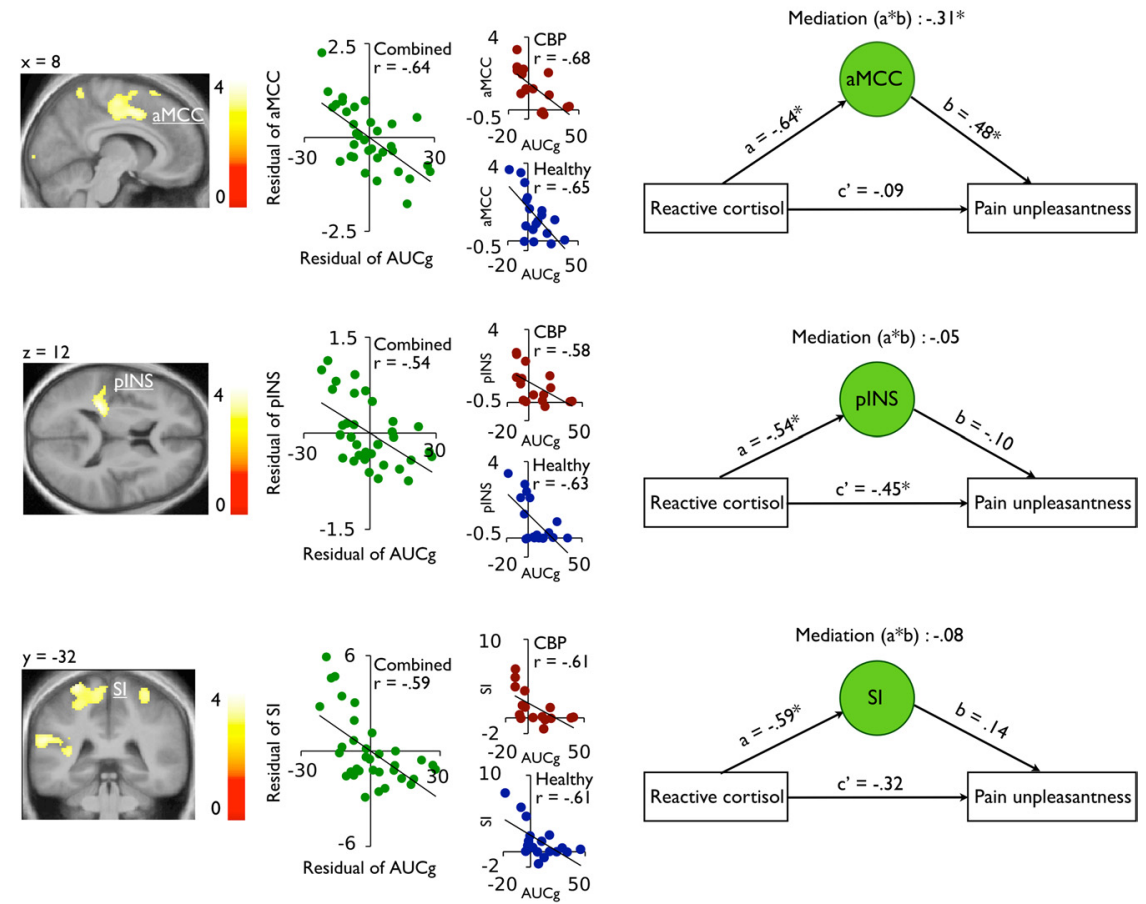

Figure 5. A, The AUCg of the reactive cortisol response negatively correlated with the BOLD signal response in the aMCC, the S1, and the pINS. The scatter plot (green) represents the residual of the mean parameter estimates (a.u.) in the each of these regions against the residual AUCg of the reactive cortisol. Additional scatter plots represent the relation between AUCg and brain activity for the CBP patients (red) and healthy individuals (blue) separately. Functional data are shown over the mean structural image of all participants (displayed at $p<0.001$ uncorrected). $\boldsymbol{B}$, Bootstrap analyses shows that the aMCC, but not $\mathrm{S} 1$ or the pINS, mediated the inverse relation between the reactive cortisol and the pain unpleasantness; ${ }^{*} p<0.05$.

\section{Discussion}

In this study, we examined the association between the individual reactive cortisol response to noxious stimuli administered in an MRI environment and pain perception and pain-related brain activity in CBP patients and healthy individuals. Consistent with the notion that pain imaging studies can be conceived as an acute stressor, the results confirmed an increase of cortisol during the 
Table 2. Negative regression of AUCg over [Pain vs Warm] with a whole brain cluster analysis

\begin{tabular}{lrrrl}
\hline & \multicolumn{3}{c}{ Local peak MNI coordinates } & \\
\cline { 2 - 4 } Brain area & $x$ & $y$ & $z$ & Local peak z-value \\
\hline Pre central gyrus & 0 & 6 & 64 & 3.42 \\
& 20 & 0 & 64 & 3.81 \\
aMCC & 10 & 0 & 46 & 3.69 \\
& 10 & -18 & 44 & 3.55 \\
Superior frontal gyrus, lateral part & -26 & 0 & 66 & 3.46 \\
& 22 & 0 & 72 & 3.60 \\
Paracentral lobule & -28 & -12 & 68 & 3.91 \\
& -4 & -18 & 52 & 3.65 \\
Post central gyrus & 18 & -50 & 68 & 3.89 \\
& -16 & -54 & 70 & 3.67 \\
Supramarginal gyrus & 18 & -50 & 68 & 3.89 \\
& -16 & -54 & 70 & 3.67 \\
Posterior insula & -30 & -32 & 14 & $3.80^{*}$
\end{tabular}

Significant clusters corrected for multiple comparisons (FWE $p<0.05$ ). ${ }^{*}$ Significant when correcting for multiple comparison in a region of interest delineating the bilateral insula (FWE $<0.05 ; p=0.02$ ).

Table 3. Psychophysical interaction on the aMCC that is decreased by stress in ROI delineating the midbrain, the pons and the medulla

\begin{tabular}{lllll}
\hline & \multicolumn{3}{l}{ Local peak MNI coordinates } & \\
\cline { 2 - 4 } Brain area & $x$ & $y$ & $z$ & Local peak $z$-value \\
\hline RVM & 0 & -34 & -50 & 3.42 \\
Midbrain & 0 & -24 & -22 & $2.78^{*}$ \\
\hline
\end{tabular}

Significant peak of activity in the RVM when corrected for multiple comparisons in a ROI delineating the midbrain, the pons and the medulla (FWE $p<0.05) ;{ }^{*} p=0.003$.

\section{Pain-related connectivity between aMCC and brainstem relative to $A \cup C g$}
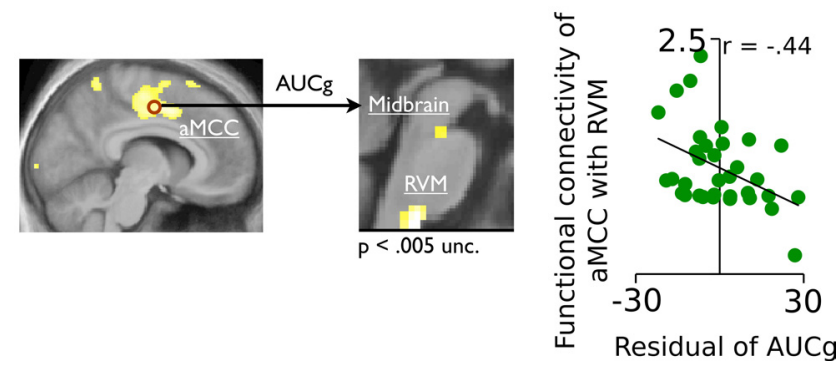

Figure 6. The functional connectivity of the aMCC with the midbrain and the RVM increased during pain, but this effect was reduced in individuals showing stronger acute stress response (AUCg). Functional data are shown over the mean structural image of all participants (displayed at $p<0.001$ uncorrected unless otherwise specified).

MRI session in both groups when compared with their basal levels. When controlling for the group and for baseline individual differences in pain sensitivity, the results indicate that individuals showing the largest reactive cortisol response reported less pain unpleasantness during scanning. This was parallel by the brain imaging results showing weaker NAc activation during the onset phase of the noxious stimulation and weaker responses in the aMCC, the pINS, and S1 during the pain stimulus. Complementary analyses established that both groups contributed to this effect. Mediation analysis permitted to determine that activation in the aMCC, but not in other pain-activated regions, mediated the inverse relation between acute stress response and pain. Interestingly, stronger stress responses also decreased the functional connectivity of the aMCC with the midbrain and the medulla. These findings are novel and important for two reasons. First, they demonstrate the association and the potential brain mediator of the relation between acute stress response and the pain experience observed in healthy and CBP patients. Second, they reveal the importance of individual acute stress responses as a major source of variability (and potential confound) in the brain response to noxious stimuli and the experience of pain.

Stress-induced analgesia has been largely documented in animal studies and is believed to result from the downregulation of neural activity in the dorsal horn of the spinal cord (Butler and Finn, 2009). Little is known, however, about the impact of acute stress on brain networks underlying the perception of pain. The neurons from the dorsal horn neurons project to supraspinal structures producing robust response in a brain network including S1-S2, the aMCC, and the insular cortex (Apkarian et al., 2005; Duerden and Albanese, 2013), which generates a cortical signature of acute pain. Several studies have shown that pain regulation consistently modulates brain activity in these areas (Price, 2000). Differences in the pain response in at least part of this brain network are also believed to explain individual variability in pain sensitivity (Coghill et al., 2003). Accordingly, our findings indicate that individuals with stronger reactive cortisol responses engaged pain-related inhibitory processes reducing brain activity in these regions.

When testing the indirect effect of brain activity on the relation between acute stress and analgesia, our results show that the association between stress responses and lower pain unpleasantness was mediated by neural activity in the aMCC, but not in S1 or the pINS. This is in accordance with a whole body of literature showing that the aMCC is a key structure involved in negative emotions, pain processing, and cognitive control (Shackman et al., 2011). It has been suggested that the aMCC could be a hub where pain and aversive events are associated to motor responses and instrumental behaviors as part of its general role in adaptation (Dum et al., 2009; Shackman et al., 2011). Consistent with this, the aMCC has connections with other limbic structures such as the amygdala (Morecraft et al., 2007), the NAc (Williams and Goldman-Rakic, 1998), and the periaqueductal gray matter (PAG; An et al., 1998) and is involved in the motivational and affective dimensions of the pain experience (Rainville et al., 1997; Johansen et al., 2001). Interestingly, previous studies have associated the acute stress measured by reactive cortisol with the reduction of activation in the anterior cingulate cortex and the limbic system during a psychological stressor (Wang et al., 2005; Pruessner et al., 2008). This suggests that the aMCC plays a critical role in the adaptation to pain and that its modulation by acute stress could be responsible for the reduction of pain unpleasantness.

Animal studies have revealed that the mechanisms of pain regulation by stress involve a combination of catecholamines (Joëls et al., 2011) and endogenous opioids (Willer et al., 1981; Akil et al., 1986), which modulate the nociceptive response of dorsal horn neurons and that these descending regulatory mechanisms are partly governed by the PAG and the rostral ventromedial medulla (RVM) (Butler and Finn, 2009). Building on this literature, we show that stress responses reduced the functional connectivity between pain-related activity in the aMCC with the midbrain and the RVM. Although the peak of activity in the midbrain was located adjacent to the PAG, these findings are consistent with animal studies and support the conception that stress activates descending modulatory mechanisms. Hence, the reduction of functional connectivity observed in our study could reflect a reduction in facilitatory activation leading to reduced ascending nociceptive signals and pain ratings. Reciprocally, the reduction in pain may reflect higher order processes leading to a reduction in pain-related brain activity in the aMCC which in turn may diminish its interaction with descending modulation 
systems. Further studies should clarify this effect by including additional measures of spinal nociceptive activity to investigate more directly the engagement of cerebrospinal regulatory processes (Piché et al., 2009; Roy et al., 2009).

Our results may also reflect a direct modulator effect of stress on cerebral activity. For instance, fMRI combined to pharmacological manipulations in humans showed that acute stress induces the release of noradrenaline, which influences large-scale networks involved in the state of arousal and the processing of sensory information (Hermans et al., 2011). Moreover, the impact of hydrocortisone has been shown on the prefrontal cortex and the hippocampus during memory formation (Henckens et al., 2012), the amygdala during the observation of fearful faces expressions (Henckens et al., 2010), and the dorsolateral prefrontal cortex during working memory (Henckens et al., 2011). These studies suggest that stress mediators promote the survival of the organism by facilitating and/or inhibiting cerebral networks involved in the function being examined in the corresponding behavioral tasks, and that these changes can be tracked by BOLDsignal changes in the corresponding brain areas. Consistent with these studies, our results suggest that the cerebral network responding to noxious stimulations is sensitive to acute stress and that the stress mediators downregulated the brain activity in pain-related areas.

A recent study provided conflicting results showing that stress induction was associated with higher pain ratings and lower pain threshold (Choi et al., 2012). Their methodology, however, only provided an after effect of stress, while our results reflect a concurrent effect of stress on pain. This is an important experimental difference because the glucocorticoids exert a rapid modulation of the synaptic plasticity followed by a long-term gene-mediated inhibition of brain plasticity favoring recovery following a stressful event (Joëls et al., 2011). Hence, it has been suggested that the rapid effects of noradrenaline interacting with the nongenomic effects of glucocorticoids last $<30 \mathrm{~min}$ while the slower genomic effect of glucocorticoids last several hours (for review, see Joëls et al., 2011). The discrepancy between the studies may therefore result from the time-dependent dynamic nature of stress responses with the rapid effects contributing to the immediate adaptation to a threatening situation and the slower effects promoting homeostatic restorative functions (de Kloet et al., 2005; Henckens et al., 2010).

On the other hand, another previous study has shown that noxious stimulation administered after a stress-induction task were rated as less painful but associated with increasing brain activity in pain responsive brain regions (i.e., S1, S2, ACC and bilateral anterior insula) (Yilmaz et al., 2010). Our findings are in sharp contrast to these results since we found a reduction of pain-related brain activity in response to thermal pain stimulations. This discrepancy might result from different methodology because we quantified the stress response by cortisol measures (basal and reactive) and we avoided using a psychological stressor adding to the already stressful MRI context (Tessner et al., 2006). Moreover, the use of psychological stress induction made the counterbalancing of experimental conditions impossible and the interpretation somehow speculative. Since most previous pain studies demonstrate that analgesia reflects a reduction of brain activations in the areas receiving projections from the spinothalamic tract (STT; Bantick et al., 2002; Wager et al., 2004; Koyama et al., 2005; Bingel et al., 2006; Piché et al., 2009; Roy et al., 2009; Villemure and Bushnell, 2009; Longo et al., 2012), we feel confident that our brain imaging results do reflect analgesic effects associated with the stress response.
One of the goals of the present study was to determine whether acute stress differently impacts the pain-related brain network in CBP patients when compared with healthy individuals. In the present study, we did not observe group differences in reactive salivary cortisol, pain perception, and pain-related brain activity. Moreover, both groups had their pain-related brain activity reduced when the individual displayed increases in reactive cortisol responses. These findings suggest that the acute stress reactivity and its impact on pain sensitivity were preserved in CBP patients. In a companion study, however, we reported group differences in basal cortisol, which supported a dysregulation of allostasis functions in CBP patients (Borsook et al., 2012; Vachon-Presseau et al., 2013). Together, these studies strengthen the predominant view that chronic pain does not disrupt the acute response to stress and the sensory dimension of pain, but induces long-term changes in neural systems underlying affective-motivational functions (Apkarian et al., 2009; Baliki et al., 2010, 2012).

\section{Conclusion}

This study shows that stronger reactive stress responses measured by cortisol are associated with reduced cerebral processing of acute noxious stimuli and result in a diminution of pain unpleasantness. More precisely, we show that reduced pain activation within the aMCC mediated the inverse relation between acute stress and pain. Further studies are, however, needed to disentangle the relative contribution of spinal and cortical processing during stress-induced analgesia in humans and determine more precisely how stress-related physiological processes contribute to chronic pain conditions.

\section{References}

Akil H, Young E, Walker JM, Watson SJ (1986) The many possible roles of opioids and related peptides in stress-induced analgesia. Ann N Y Acad Sci 467:140-153. CrossRef Medline

An X, Bandler R, Ongür D, Price JL (1998) Prefrontal cortical projections to longitudinal columns in the midbrain periaqueductal gray in macaque monkeys. J Comp Neurol 401:455-479. CrossRef Medline

Apkarian AV, Bushnell MC, Treede RD, Zubieta JK (2005) Human brain mechanisms of pain perception and regulation in health and disease. Eur J Pain 9:463-484. CrossRef Medline

Apkarian AV, Baliki MN, Geha PY (2009) Towards a theory of chronic pain. Prog Neurobiol 87:81-97. CrossRef Medline

Baliki MN, Geha PY, Fields HL, Apkarian AV (2010) Predicting value of pain and analgesia: nucleus accumbens response to noxious stimuli changes in the presence of chronic pain. Neuron 66:149-160. CrossRef Medline

Baliki MN, Petre B, Torbey S, Herrmann KM, Huang L, Schnitzer TJ, Fields HL, Apkarian AV (2012) Corticostriatal functional connectivity predicts transition to chronic back pain. Nat Neurosci 15:1117-1119. CrossRef Medline

Bantick SJ, Wise RG, Ploghaus A, Clare S, Smith SM, Tracey I (2002) Imaging how attention modulates pain in humans using functional MRI. Brain 125:310-319. CrossRef Medline

Becerra L, Breiter HC, Wise R, Gonzalez RG, Borsook D (2001) Reward circuitry activation by noxious thermal stimuli. Neuron 32:927-946. CrossRef Medline

Beck AT, Steer RA, Brown GK (1996) Manual for the beck depression inventory-II. San Antonio, TX: Psychological Corporation.

Bingel U, Lorenz J, Schoell E, Weiller C, Büchel C (2006) Mechanisms of placebo analgesia: $\mathrm{AACC}$ recruitment of a subcortical antinociceptive network. Pain 120:8-15. CrossRef Medline

Bornhövd K, Quante M, Glauche V, Bromm B, Weiller C, Büchel C (2002) Painful stimuli evoke different stimulus-response functions in the amygdala, prefrontal, insula and somatosensory cortex: a single-trial fMRI study. Brain 125:1326-1336. CrossRef Medline

Borsook D, Maleki N, Becerra L, McEwen B (2012) Understanding mi- 
graine through the lens of maladaptive stress responses: a model disease of allostatic load. Neuron 73:219-234. CrossRef Medline

Butler RK, Finn DP (2009) Stress-induced analgesia. Prog Neurobiol 88: 184-202. CrossRef Medline

Chen AC, Dworkin SF, Haug J, Gehrig J (1989) Human pain responsivity in a tonic pain model: psychological determinants. Pain 37:143-160. CrossRef Medline

Choi JC, Chung MI, Lee YD (2012) Modulation of pain sensation by stressrelated testosterone and cortisol. Anaesthesia 67:1146-1151. CrossRef Medline

Coghill RC, McHaffie JG, Yen YF (2003) Neural correlates of interindividual differences in the subjective experience of pain. Proc Natl Acad Sci U S A 100:8538-8542. CrossRef Medline

de Kloet ER, Joëls M, Holsboer F (2005) Stress and the brain: from adaptation to disease. Nat Rev Neurosci 6:463-475. CrossRef Medline

Dionne RA, Bartoshuk L, Mogil J, Witter J (2005) Individual responder analyses for pain: does one pain scale fit all? Trends Pharmacol Sci 26:125130. CrossRef Medline

Duerden EG, Albanese MC (2013) Localization of pain-related brain activation: a meta-analysis of neuroimaging data. Hum Brain Mapp 34:109149. CrossRef Medline

Dum RP, Levinthal DJ, Strick PL (2009) The spinothalamic system targets motor and sensory areas in the cerebral cortex of monkeys. J Neurosci 29:14223-14235. CrossRef Medline

Friedman SB, Ader R, Grota LJ, Larson T (1967) Plasma corticosterone response to parameters of electric shock stimulation in the rat. Psychosom Med 29:323-328. Medline

Friston KJ, Buechel C, Fink GR, Morris J, Rolls E, Dolan RJ (1997) Psychophysiological and modulatory interactions in neuroimaging. Neuroimage 6:218-229. CrossRef Medline

Henckens MJ, van Wingen GA, Joëls M, Fernández G (2010) Timedependent effects of corticosteroids on human amygdala processing. J Neurosci 30:12725-12732. CrossRef Medline

Henckens MJ, van Wingen GA, Joëls M, Fernández G (2011) Timedependent corticosteroid modulation of prefrontal working memory processing. Proc Natl Acad Sci U S A 108:5801-5806. CrossRef Medline

Henckens MJ, Pu Z, Hermans EJ, van Wingen GA, Joëls M, Fernández G (2012) Dynamically changing effects of corticosteroids on human hippocampal and prefrontal processing. Hum Brain Mapp 33:2885-2897. CrossRef Medline

Hermans EJ, van Marle HJ, Ossewaarde L, Henckens MJ, Qin S, van Kesteren MT, Schoots VC, Cousijn H, Rijpkema M, Oostenveld R, Fernández G (2011) Stress-related noradrenergic activity prompts large-scale neural network reconfiguration. Science 334:1151-1153. CrossRef Medline

Hohmann AG, Suplita RL, Bolton NM, Neely MH, Fegley D, Mangieri R, Krey JF, Walker JM, Holmes PV, Crystal JD, Duranti A, Tontini A, Mor M, Tarzia G, Piomelli D (2005) An endocannabinoid mechanism for stress-induced analgesia. Nature 435:1108-1112. CrossRef Medline

Joëls M, Fernandez G, Roozendaal B (2011) Stress and emotional memory: a matter of timing. Trends Cogn Sci 15:280-288. CrossRef Medline

Johansen JP, Fields HL, Manning BH (2001) The affective component of pain in rodents: direct evidence for a contribution of the anterior cingulate cortex. Proc Natl Acad Sci U S A 98:8077-8082. CrossRef Medline

Kirschbaum C, Hellhammer DH (1994) Salivary cortisol in psychoneuroendocrine research: recent developments and applications. Psychoneuroendocrinology 19:313-333. CrossRef Medline

Koyama T, McHaffie JG, Laurienti PJ, Coghill RC (2005) The subjective experience of pain: where expectations become reality. Proc Natl Acad Sci U S A 102:12950-12955. CrossRef Medline

Lleras C (2005) Path analysis. In: Encyclopedia of social measurement (Kempf-Leonard K, ed), pp 25-30. New-York: Academic.

Longo MR, Iannetti GD, Mancini F, Driver J, Haggard P (2012) Linking pain and the body: neural correlates of visually induced analgesia. J Neurosci 32:2601-2607. CrossRef Medline

Madden J 4th, Akil H, Patrick RL, Barchas JD (1977) Stress-induced parallel changes in central opioid levels and pain responsiveness in the rat. Nature 265:358-360. CrossRef Medline
Maldjian JA, Laurienti PJ, Kraft RA, Burdette JH (2003) An automated method for neuroanatomic and cytoarchitectonic atlas-based interrogation of fMRI data sets. Neuroimage 19:1233-1239. CrossRef Medline

Melzack R (1975) The McGill Pain Questionnaire: major properties and scoring methods. Pain 1:277-299. CrossRef Medline

Morecraft RJ, McNeal DW, Stilwell-Morecraft KS, Gedney M, Ge J, Schroeder CM, van Hoesen GW (2007) Amygdala interconnections with the cingulate motor cortex in the rhesus monkey. J Comp Neurol 500:134-165. CrossRef Medline

Nielsen CS, Staud R, Price DD (2009) Individual differences in pain sensitivity: measurement, causation, and consequences. J Pain 10:231-237. CrossRef Medline

Piché M, Arsenault M, Rainville P (2009) Cerebral and cerebrospinal processes underlying counterirritation analgesia. J Neurosci 29:14236-14246. CrossRef Medline

Price DD (2000) Psychological and neural mechanisms of the affective dimension of pain. Science 288:1769-1772. CrossRef Medline

Pruessner JC, Kirschbaum C, Meinlschmid G, Hellhammer DH (2003) Two formulas for computation of the area under the curve represent measures of total hormone concentration versus time-dependent change. Psychoneuroendocrinology 28:916-931. CrossRef Medline

Pruessner JC, Dedovic K, Khalili-Mahani N, Engert V, Pruessner M, Buss C, Renwick R, Dagher A, Meaney MJ, Lupien S (2008) Deactivation of the limbic system during acute psychosocial stress: evidence from positron emission tomography and functional magnetic resonance imaging studies. Biol Psychiatry 63:234-240. CrossRef Medline

Rainville P, Duncan GH, Price DD, Carrier B, Bushnell MC (1997) Pain affect encoded in human anterior cingulate but not somatosensory cortex. Science 277:968-971. CrossRef Medline

Roy M, Piché M, Chen JI, Peretz I, Rainville P (2009) Cerebral and spinal modulation of pain by emotions. Proc Natl Acad Sci U S A 106:20900-20905. CrossRef Medline

Shackman AJ, Salomons TV, Slagter HA, Fox AS, Winter JJ, Davidson RJ (2011) The integration of negative affect, pain and cognitive control in the cingulate cortex. Nat Rev Neurosci 12:154-167. CrossRef Medline

Tessner KD, Walker EF, Hochman K, Hamann S (2006) Cortisol responses of healthy volunteers undergoing magnetic resonance imaging. Hum Brain Mapp 27:889-895. CrossRef Medline

Vachon-Presseau E, Roy M, Martel MO, Albouy G, Chen J, Budell L, Sullivan MJ, Jackson PL, Rainville P (2012) Neural processing of sensory and emotional-communicative information associated with the perception of vicarious pain. Neuroimage 63:54-62. CrossRef Medline

Vachon-Presseau E, Roy M, Martel MO, Caron E, Marin MF, Chen J-I, Albouy G, Plante I, Sullivan MJ, Lupien SJ, Rainville P (2013) The stress model of chronic pain: evidence from basal cortisol and hippocampal structure and function in humans. Brain 136:815-827.

Villemure C, Bushnell MC (2009) Mood influences supraspinal pain processing separately from attention. J Neurosci 29:705-715. CrossRef Medline

Wager TD, Rilling JK, Smith EE, Sokolik A, Casey KL, Davidson RJ, Kosslyn SM, Rose RM, Cohen JD (2004) Placebo-induced changes in FMRI in the anticipation and experience of pain. Science 303:1162-1167. CrossRef Medline

Wang J, Rao H, Wetmore GS, Furlan PM, Korczykowski M, Dinges DF, Detre JA (2005) Perfusion functional MRI reveals cerebral blood flow pattern under psychological stress. Proc Natl Acad Sci U S A 102:17804-17809. CrossRef Medline

Willer JC, Dehen H, Cambier J (1981) Stress-induced analgesia in humans: endogenous opioids and naloxone-reversible depression of pain reflexes. Science 212:689-691. CrossRef Medline

Williams SM, Goldman-Rakic PS (1998) Widespread origin of the primate mesofrontal dopamine system. Cereb Cortex 8:321-345. CrossRef Medline

Yilmaz P, Diers M, Diener S, Rance M, Wessa M, Flor H (2010) Brain correlates of stress-induced analgesia. Pain 151:522-529. CrossRef Medline 